\title{
Korean medicine students' entrance factors and satisfaction with their college experience: a quantitative nationwide study
}

Hyun-Seok Kim

Dongshin University College of Korean Medicine Joohyun Lee

Dongshin University College of Korean Medicine

Hwimun Kim

Dongshin University College of Korean Medicine

Unho Yang

Dongshin University College of Korean Medicine

Hyunho Kim ( $\nabla$ hyunho.kim@cymedical.org )

Research article

Keywords: entrance factor, premedical education, traditional Korean medicine, college satisfaction

Posted Date: April 13th, 2020

DOI: https://doi.org/10.21203/rs.2.20049/v3

License: (1) (i) This work is licensed under a Creative Commons Attribution 4.0 International License.

Read Full License 


\section{Abstract}

Background: Korean medicine (KM) has equal legal rights with conventional Western medicine in South Korea and is taught at KM colleges. To date, no research has been conducted on the entrance factors and satisfaction of KM college students. Thus, this study attempts to fill this gap in the literature.

Methods: Content validity tests and face validity tests were conducted during the development of the questionnaires used in this study. The scores of each item and the correlation between items and subitems were analyzed and factors were correlated with students' satisfaction and willingness to re-enter $\mathrm{KM}$ colleges.

Results: A total of 420 students responded to the survey. The means of the items considering stable incumbency and examination scores were the highest. The highest correlation between an item and subitem concerned interaction with people. The questionnaire items or sub-questionnaire items that showed moderate high correlation with the questionnaire items asked whether participants were willing to re-enter the KM colleges and were both about independent choices.

Conclusions: Identifying college entrance factors can contribute to increasing current students' academic satisfaction and decreasing drop-out rates. This study highlights factors that can be applied in college curriculum or subject teaching, such as interest in research on KM.

\section{Background}

Students entering college choose their major based on various factors. Their perception of their major affects their satisfaction with the curriculum, and their perception of their department affects their satisfaction during enrollment and academic adjustment [1]. Factors such as students' individual characteristics, values, and needs [2] affect their medical school experience. Prior research has shown that the factors affecting medical students' choices of majors are becoming increasingly complex $[3,4]$. Therefore, identifying relevant college entrance factors is important for establishing healthcare provisions because these factors reflect students' perspectives on their compatibility with certain fields and the flexibility of various jobs [3].

Furthermore, students' primary career plans may change over the course of their college education and are influenced by the degree to which college education presents diverse career opportunities [2]. Therefore, medical students' major choice or satisfaction level may differ according to their motivation for entering college. These factors may not only impact students' career decisions, but also those of policy makers and educators.

Many researchers have examined college entrance factors, as they have a high impact on the healthcare system. The results of related research have reported factors that make students likely to become doctors, college entrance factors according to gender, and college entrance factors that differ between medical students and dental students [5-8]. 
Korean medicine $(\mathrm{KM})$, also known as traditional Korean medicine, is distinct from conventional Western medicine but has equal legal rights in South Korea's healthcare system. Korean medicine is based on traditional Chinese medicine but encompasses unique practices such as four-constitution medicine and saam acupuncture [9]. Korean medicine considers human beings as holistic entities and employs both a modern medical diagnosis system and a unique pattern diagnosis theory [10]. Korean medicine doctors (KMD) use codes from the Korean Standard Classification of Disease 7th edition (KCD7) to diagnose, prescribe, and select the most appropriate description of a patient's condition. This code is based on International Classification of Diseases 10th Revision(ICD-10) and includes the original disease codes of $\mathrm{KM}$. These original codes for Korean medicine contributed to traditional medicine conditions-Module 1 , included in ICD-11 [9].

The education curriculum of many KM colleges is a six-year program consisting of two years of premedical school and four years of medical school [11]. The purpose of KM colleges is to cultivate KMD who have learned about the traditions of KM, possess both scientific knowledge and clinical skills, and volunteer their knowledge in society [12]. To meet this purpose, KM colleges provide a balanced education in modern biomedicine as well as traditional KM knowledge $[9,13]$. The Institute of Korean Medicine Education and Evaluation was established to ensure quality control in KM education. Because of the distinct characteristics of KM colleges, the education provided by such colleges has been evaluated from various perspectives such as the level of satisfaction of KM college students, their education in evidencebased medicine, and the introduction of problem-based learning [14-16].

However, factors that influence KM college entrance and motivation have not been evaluated in previous studies. Further, students of KM colleges only share some characteristics with medical and dental students; therefore, it is necessary to examine the college entrance factors of KM college students independently. Evaluating such factors may allow the identification of potential KM students in the course of career guidance. Furthermore, some factors that are significantly related to students' satisfaction with KM college life can be used to establish constructive KM educational environments. Therefore, the purpose of this study is to identify the factors that induce KM college students to attend $\mathrm{KM}$ colleges and the factors that are significantly related to their satisfaction levels with their colleges.

\section{Methods}

\section{Designing questionnaire items}

To design the questionnaire items for KM students, we followed the questionnaire design guideline from AMEE (An International Association for Medical Education) [17]. The guideline mainly consists of the process of collecting and synthesizing literature review, item developing, content validity test, cognitive interviewing, and pre-testing [17]. Based on the results of questionnaires conducted on medical, dental, and KM college students $[5,7,18]$ and discussions between the authors of this study, seven latent variables were defined: (1) status and security (social status, stable incumbency, expecting higher earning), (2) career (research, clinical activities), (3) patient care and working with people (interactions 
with people), (4) requiring personal and clinical skills (manual skills, psychological skills), (5) interest in $\mathrm{KM}$ (interest in the theory of KM, interest in KM treatment methods based on modern science), (6) personal experience or atmosphere (positive experience receiving KM treatment, military duty, examination scores, belief that KM is students' calling, chronic disease experienced by the student or their family member), (7) influencing person or method (family and relatives, acquaintances, mass media, independent choice).

To conduct a general investigation of students' satisfaction levels, we created two questionnaire items: 'I am satisfied with my current enrollment in the college/graduate school of Korean medicine' and 'If I were to restart my post-secondary education, I would choose the college/graduate school of Korean medicine again.'

We constructed questionnaires to obtain demographic information including participants' age; gender; whether they were currently attending university; whether they had a doctor, dentist, or Korean medicine doctor in their family including parents and siblings; and their academic discipline group. The questionnaire was used to examine college-related factors rather than respondents' general perceptions of $\mathrm{KM}$. Therefore, examples of the questionnaire items are included to provide a better understanding of participants' answers.

The twenty-two questionnaire items described above were constructed with a 5-point Likert scale. However, because some participants were female or had already finished their military service, the questionnaire item related to 'military duty' included an additional 'not applicable' answer. The study intended to identify KM college students' satisfaction levels both at the point they decided to matriculate and at the present time. Thus, in addition to the two questionnaire items stating 'I am satisfied with my current enrollment in the college/graduate school of Korean medicine' and 'If I were to restart my postsecondary education, I would choose the college/graduate school of Korean medicine again', a subquestionnaire item stating 'My experience attending this school is satisfactory for the same reason as above' was included.

\section{Content validity test}

Content validity determines whether the developed questionnaire items properly reflect the content [19]. In this study, the researchers measured I-CVI, which involves the representativeness of individual questionnaire items [20]. Content experts measured the representativeness of individual items with a 4point Likert scale, after which the researchers divided the number of experts who rated each item as three or four by the total number of experts. Items with an average I-CVI score of 0.8 or higher were regarded as content valid $[19,20]$. Content experts are those who conduct research in the relevant field or who have experience in that field [20]. Therefore, for our research, we selected three experts: a KM college professor teaching basic science, a KM college professor teaching clinical medicine, and a professor who had major experience in survey research. Furthermore, we selected three consultants for college entrance. 
After acquiring consent from the six experts, one of the researchers explained the method and implication of the content validity measurement. Experts were given a panel link created with Google Forms (www.google.com/forms, Mountain View, CA, USA), an online survey platform. The representativeness, clarity, and relativeness of each item consisting of each latent variable were measured by evaluating items with a score range of one to four. To acquire meaningful responses from experts [21], qualitative data about each latent variable or each item were collected.

By reviewing the qualitative data of items with 0.8 or higher I-CVI, 16 items identified for revision were edited, and the content validity experts were asked to check whether the edited items represented their content well. Items with I-CVI lower than 0.8 were items about 'manual skills' and 'psychological skills,' and their I-CVI were 0.5 each. These questionnaire items were edited by reviewing the qualitative data first, after which the representativeness, clarity, and relativeness of individual items were measured with a 4-point Likert scale again. Through additional comments from experts, the item 'potential for future development of KM' was added as a latent variable about participants' interest in Korean medicine. The item 'Making policies and performing social activities related to KM' was added as a latent variable regarding careers. The representativeness, clarity, and relativeness of the new questionnaire items were then measured with a 4-point Likert scale. Therefore, a total of 24 questionnaire items were created.

\section{Face validity and pre-test}

Measuring face validity involves asking people who are related to a subject group whether the questions appear to be valid and gathering the respondents' oral opinions about the questionnaire [22]. Measurement for assessing face validity can include cognitive interview process [23]. First and second year medical students were selected as participants for face validity tests as they had experienced premedical second year. One group of seven medical first year students and another group of six medical second year students participated in focus group interviews. The coding criteria in the interviews examined the proper implementation of the researchers' intentions [24]. Therefore, the researcher introduced the intention of each questionnaire item to the groups and let them freely discuss their feelings. The process of face validity includes identifying and editing questionnaire items that participants have difficulty understanding, and checking whether they understand the edited questionnaire items. Through this process, the example written in the survey was edited for better understanding. Questionnaire items about 'examination scores' were also edited from 'because of the examination scores' to 'because examination scores are an important factor.' The final online survey questionnaire is described in Additional File 1 (Additional file 1). Before the survey, a pre-test was conducted with premedical second year students.

\section{Subjects and Survey Procedure}

In the KM curriculum, students are separated into the premedical and medical levels. Premedical second year is a sophomore year in which students learn basic KM theory, basic medical science, and liberal arts. Considering recall bias, the survey did not target upper grades. As premedical first year students are freshmen, the researchers assumed that they do not have enough experience of KM colleges; therefore, 
premedical second year students were selected as research participants. As there are several curriculum differences between each KM college, research was conducted among all KM colleges in South Korea, with a total of 744 students. The survey was conducted over ten days, from April 8 to April 17, 2019. The online survey link was distributed via a message to student group leaders of each KM college's premedical second year students. Pre-participants were informed of the following before participating in the study: background and aim of the research, type of participants, methods, dates of the study, benefits from participating in the research, lack of direct side-effects, rewards or costs, privacy of personal information, voluntary participation and how to end their participation, and contact details. Only participants who read this information and gave their informed consent online could participate in the study.

\section{IRB approval and informed consent}

This study was conducted after receiving approval from the Public Institutional Bioethics Committee designated by the MOHW (P01-201903-22-003). Personal information acquired by the study was protected using methods provided by the online survey platform SurveyMonkey ${ }^{\mathrm{TM}}$

(www.surveymonkey.com, LLC; Palo Alto, CA, USA) and IRB institution. The personal information collected consisted only of a cellular phone number that was used to provide participants with a $\$ 1$ reward. Participants were informed about the collection of this information before they took the survey. Those who wanted to stop participating were free to do so at any time. To this end, the online survey provided two researchers' phone numbers and e-mail addresses. After the survey was conducted and rewards were distributed, the personal information was completely destroyed.

\section{Data analysis and statistical analysis}

Responses from 420 students who participated in the survey among the $774 \mathrm{KM}$ students were analyzed. The response rates and percentage of each response were described as well as the number of participants and response rates (ratio of participating students to total enrollment of KM colleges' premedical second year) of each KM college. Frequency, mean, and standard deviation (SD) of questionnaire items were analyzed on a 5-point Likert scale. To identify the correlation between entrance factors and present satisfactory levels, a Pearson's correlation test of questionnaire items and subquestionnaire items was conducted. To determine the factors that affect KM college students' general level of satisfaction, a Pearson's correlation test was conducted between the questionnaire item 'I am satisfied with my current enrollment in the college/graduate school of Korean medicine' and the questionnaires of the five latent variables, as well as the questionnaire item 'If I were to restart my postsecondary education, I would choose the college/graduate school of Korean medicine again' and the questionnaires of the five latent variables. The programs used for statistical analysis were Excel 2013 (Microsoft, Redmond, WA, USA), IBM SPSS Statistics 25 (SPSS Inc., Chicago, IL, USA) and Graphpad Prism 7 (GraphPad ${ }^{\text {TM }}$ Software Inc., San Diego, California, USA).

\section{Results}


Content validity index 
Table 1 I-CVI Score of Each Item

Title of Questionnaire Items

Experts

I-

CVI

1. I entered the college/graduate school of Korean medicine because I think

\begin{tabular}{llllll}
\hline & $\square$ & $\square$ & $\square$ & $\square$ & $\square$
\end{tabular}

3

Korean medicine doctors are professionals with high social status.

$\begin{array}{lllllll}3 & 4 & 4 & 4 & 4 & 4 & 1.0\end{array}$

3

1. I entered the college/graduate school of Korean medicine because I think

$\begin{array}{lllllll}3 & 4 & 4 & 4 & 4 & 4 & 1.0\end{array}$

Korean medicine doctors are professionals with a stable incumbency.

1. I entered the college/graduate school of Korean medicine because I expect

$\begin{array}{lllllll}3 & 4 & 4 & 4 & 4 & 4 & 1.0\end{array}$

high earnings as a doctor of Korean medicine.

1. I entered the college/graduate school of Korean medicine because I am $\begin{array}{lllllll}3 & 4 & 3 & 4 & 4 & 4 & 1.0\end{array}$ interested in the theory of Korean medicine.

1. I entered the college/graduate school of Korean medicine because my experience or my family and my acquaintances' experience receiving Korean medicine treatment was positive.

1. I entered the college/graduate school of Korean medicine because I am interested in treatment methods different from Western medicine.

$\begin{array}{lllllll}3 & 4 & 3 & 4 & 4 & 4 & 1.0\end{array}$

1. I entered the college/graduate school of Korean medicine because I think Korean medicine is based on modern science.

$\begin{array}{lllllll}3 & 4 & 3 & 4 & 4 & 4 & 1.0\end{array}$

$\begin{array}{lllllll}3 & 3 & 3 & 4 & 4 & 4 & 1.0\end{array}$

1. I entered the college/graduate school of Korean medicine because I would $\begin{array}{lllllll}2 & 4 & 4 & 4 & 4 & 4 & 0.83\end{array}$ like to carry out research.

1. I entered the college/graduate school of Korean medicine because I would like to perform clinical activities (opening a clinic or working at a hospital)

1. I entered the college/graduate school of Korean medicine because Korean medicine doctors interact with people.

$\begin{array}{lllllll}3 & 4 & 4 & 4 & 4 & 4 & 1.0\end{array}$

$\begin{array}{lllllll}2 & 4 & 3 & 4 & 4 & 4 & 0.83\end{array}$

1. I entered the college/graduate school of Korean medicine because I can perform manual skills as a Korean medicine doctor.

1. I entered the college/graduate school of Korean medicine because I can perform psychological skills, including counseling, as a Korean medicine doctor.

1. I entered the college/graduate school of Korean medicine because of my military duty (as a public health doctor or an army doctor).

1. I entered the college/graduate school of Korean medicine because of my college entrance examination scores (college scholastic ability test, high school records) at the time of college application.

$\begin{array}{lllllll}2 & 2 & 2 & 4 & 4 & 4 & 0.5\end{array}$

$\begin{array}{lllllll}2 & 1 & 2 & 4 & 4 & 4 & 0.5\end{array}$

1. I entered the college/graduate school of Korean medicine because I think it is 2 $\begin{array}{lllllll}3 & 4 & 3 & 4 & 4 & 4 & 1.0\end{array}$ $\begin{array}{lllllll}3 & 4 & 3 & 4 & 4 & 4 & 1.0\end{array}$ my calling. 


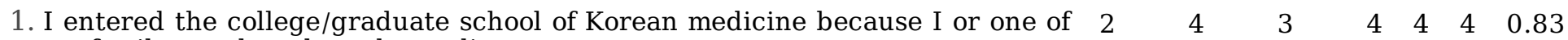
my family members have/has a disease.

$\begin{array}{lllllll}3 & 3 & 4 & 4 & 4 & 4 & 1.0\end{array}$

1. My family influenced my decision to attend the college/graduate school of Korean medicine.

1. My acquaintances (including friends, teachers, and lecturers) influenced my

$\begin{array}{lllllll}3 & 4 & 4 & 4 & 4 & 4 & 1.0\end{array}$
decision to attend the college/graduate school of Korean medicine.

1. Mass media influenced my decision to attend the college/graduate school of Korean medicine.

$\begin{array}{lllllll}3 & 4 & 3 & 4 & 4 & 4 & 1.0\end{array}$

1. I independently chose to attend the college/graduate school of Korean medicine.

$3 \quad 4 \quad 4 \quad 4 \quad 4 \quad 4 \quad 1.0$

$\begin{array}{lllllllllll} & 3 & 4 & 4 & 4 & 4 & 4 & 1.0\end{array}$

. I currently satisfied with my enrollment in the college/graduate school of Korean medicine.

1. If I were to restart my post-secondary education, I would choose the $\begin{array}{lllllll}3 & 4 & 4 & 4 & 4 & 4 & 1.0\end{array}$ college/graduate school of Korean medicine again.
Mean
$\begin{array}{lllllll}2.73 & 3.63 & 3.36 & 4 & 4 & 4 & 0.92\end{array}$

*I-CVI: item-level content validity index **[य]: professors majored in Korean Medicine

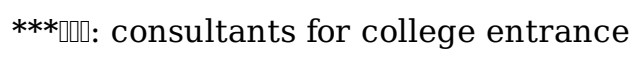

Professors who majored in KM rated questionnaire items about 'clinical skills' and 'psychological skills' relatively low (Table 1). They pointed out that 'manual skills' is an inappropriate questionnaire item that required respondents to include detailed examples. 
Table 2 I-CVI Score of New Items And Edited Questionnaire Items

Title of modified questionnaire items

(b) I entered the college/graduate school of Korean medicine because I think Korean medicine doctors are professionals with a stable incumbency.

(c) I entered the college/graduate school of Korean medicine because I expect higher earnings as a doctor of Korean medicine than other professions after graduation.

(f) I entered the college/graduate school of Korean medicine because I am interested in treatment methods found in Korean medicine different from Western medicine.

(h) I entered the college/graduate school of Korean medicine because I would like to carry out research related to Korean medicine.

(i) I entered the college/graduate school of Korean medicine because I would like to perform clinical activities (including working at a hospital or a Korean medicine clinic).

(j) I entered the college/graduate school of Korean medicine because Korean medicine requires interaction with people who need examinations and treatments to be done by Korean medicine doctors.

(m) I entered the college/graduate school of Korean medicine because I can fulfill my military duty (as a public health doctor or an army doctor) easier than other occupational groups.

(n) I entered the college/graduate school of Korean medicine because college entrance examination scores (college scholastic ability test, high school records) were important factors at the time of college application.

(o) I entered the college/graduate school of Korean medicine because I think Korean medicine doctors and the related occupational scope of practice are closely related to my calling.

(p) I entered the college/graduate school of Korean medicine because I or one of my family members have/has a chronic disease.

(q) My family and relatives influenced my decision to attend the college/graduate school of Korean medicine. .

(s) Mass media (including TV programs, the Internet, Korean medicine doctors as role models in the media) influenced my decision to attend the college/graduate school of Korean medicine.

(u) I am satisfied with my current enrollment in the college/graduate school of Korean medicine.

Title of newly added questionnaire items Experts

I-

CVI

I entered the college/graduate school of Korean medicine because I think Korean $\quad 4 \quad 4 \quad 4 \quad 3 \quad 4 \quad 4 \quad 4 \quad 4 \quad 1.0$ medicine has potential for future development.

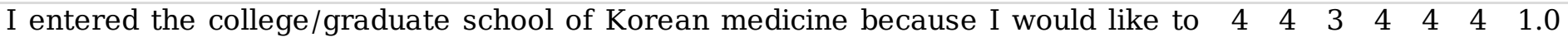
make policies and perform social activities related to Korean medicine.

(k) I entered the college/graduate school of Korean medicine because I can learn to $\quad 4 \quad 4 \quad 4 \quad 3 \quad 4 \quad 4 \quad 4 \quad 4 \quad 1.0$ perform clinical skills (including acupuncture, moxibustion, and herbal medicine) myself as a Korean medicine doctor.

(l) I entered the college/graduate school of Korean medicine because I can perform $\quad \begin{array}{llllllll}4 & 4 & 3 & 4 & 4 & 4 & 1.0\end{array}$ psychological treatments, including counseling, as a Korean medicine doctor.

*I-CVI: item-level content validity index **au: professors majored in Korean Medicine ***aा: consultants for college entrance

The questionnaire items 'perform manual skills as a Korean medicine doctor' and 'perform psychological skills' were modified to 'learn to perform clinical skills (including acupuncture, moxibustion, and herbal medicine) myself' and 'perform psychological treatments.' After the modification, I-CVI was calculated as 1.0 (Table 2). 
Two questionnaire items were developed based on qualitative data from experts, one of which states, 'I entered the college/graduate school of Korean medicine because I think Korean medicine has potential for future development,' categorized into the latent variable 'interest in Korean medicine.' The other is the item stating 'I entered the college/graduate school of Korean medicine because I would like to make policies and perform social activities related to Korean medicine,' categorized into the latent variable 'career.' The average I-CVI of the two items was 1.0 (Table 2).

Twelve questionnaire items that had 0.8 or higher I-CVI but required modifications according to qualitative data from experts were modified. After the revision, we determined whether the questionnaire items were adequate by showing the unmodified and modified questionnaire items to the experts (Table 2).

\section{Basic characteristics of respondents and overall survey results}

There were 209 male participants and 207 female participants. Of the 744 enrolled students, 420 participated. Therefore, the response rate was $56.5 \%$. Detailed characteristics of respondents are included in Additional File 2 (Additional file 2).

The highest mean for questionnaire items regarding entrance factors were related to stable incumbency (Mean: 4.08), examination scores (Mean: 4.03), clinical activities (Mean: 4.00), and military duty (Mean: 3.97). The highest mean for questionnaire items regarding students' satisfaction was related to whether they were satisfied with their current enrollment in KM college/graduate school (Mean: 3.90). The lowest mean for questionnaire items regarding entrance factors was related to chronic diseases of students or their family members (Mean: 2.18), mass media (Mean: 2.60), and the construction of policies and performance of social activities based on modern science (Mean: 2.65). The lowest mean for questionnaire items regarding students' satisfaction was related to chronic diseases of students or their family members (Mean: 2.18).

Pearson's correlation between questionnaire and sub-questionnaire items 
Table 3 Mean and Standard Deviation of Each Questionnaire Item

Number and title of questionnaire items

Mean \pm SD

1. I entered the college/graduate school of Korean medicine because I think Korean medicine doctors $3.85 \pm 0.8$ are professionals with high social status.

1-1. My experience attending this school is satisfactory for the same reason as above.

2. I entered the college/graduate school of Korean medicine because I think Korean medicine doctors $4.08 \pm 0.7$ are professionals with a stable incumbency.

2-1. My experience attending this school is satisfactory for the same reason as above.

3. I entered the college/graduate school of Korean medicine because I expect higher earnings as a $3.93 \pm 0.8$ doctor of Korean medicine than other professions after graduation.

3-1. My experience attending this school is satisfactory for the same reason as above.

4. I entered the college/graduate school of Korean medicine because I am interested in the theory of $2.99 \pm 1.1$ Korean medicine.

4-1. My experience attending this school is satisfactory for the same reason as above.

5. I entered the college/graduate school of Korean medicine because my experience or my family and $3.21 \pm 1.2$ my acquaintances' experience receiving Korean medicine treatment was positive.

5-1. My experience attending this school is satisfactory for the same reason as above.

6. I entered the college/graduate school of Korean medicine because I am interested in treatment $3.10 \pm 1.1$ methods found in Korean medicine different from Western medicine

6-1. My experience attending this school is satisfactory for the same reason as above.

$3.23 \pm 1.0$

7. I entered the college/graduate school of Korean medicine because I think Korean medicine is based $2.65 \pm 1.0$ on modern science.

7-1. My experience attending this school is satisfactory for the same reason as above.

$2.90 \pm 0.9$

8. I entered the college/graduate school of Korean medicine because I think Korean medicine has $3.48 \pm 1.0$ potential for future development.

8-1. My experience attending this school is satisfactory for the same reason as above.

$3.40 \pm 0.9$

9. I entered the college/graduate school of Korean medicine because I would like to carry out research $2.68 \pm 1.1$ related to Korean Medicine.

9-1. My experience attending this school is satisfactory for the same reason as above.

$2.92 \pm 1.0$

10. I entered the college/graduate school of Korean medicine because I would like to perform clinical $4.00 \pm 1.0$ activities (including working at a hospital or a Korean medicine clinic).

10-1. My experience attending this school is satisfactory for the same reason as above.

$3.83 \pm 0.9$

11. I entered the college/graduate school of Korean medicine because I would like to make policies $2.60 \pm 1.0$ and perform social activities related to Korean medicine.

11-1. My experience attending this school is satisfactory for the same reason as above.

$2.88 \pm 1.0$

12. I entered the college/graduate school of Korean medicine because Korean medicine requires $3.62 \pm 1.1$ interaction with people who need examinations and treatments to be done by Korean medicine doctors. 
12-1. My experience attending this school is satisfactory for the same reason as above.

13. I entered the college/graduate school of Korean medicine because I can learn to perform clinical $3.85 \pm 1.0$ skills (including acupuncture, moxibustion, and herbal medicine) myself as a Korean medicine doctor.

13-1. My experience attending this school is satisfactory for the same reason as above.

14. I entered the college/graduate school of Korean medicine because I can perform psychological $3.44 \pm 1.1$ treatments, including counseling, as a Korean medicine doctor.

14-1. My experience attending this school is satisfactory for the same reason as above.

15. I entered the college/graduate school of Korean medicine because I can fulfill my military duty (as $3.97 \pm 1.1$ a public health doctor or an army doctor) easier than other occupational groups.

15-1. My experience attending this school is satisfactory for the same reason as above.

16. I entered the college/graduate school of Korean medicine because college entrance examination $4.03 \pm 0.9$ scores (college scholastic ability test, high school records) were important factors at the time of college application.

16-1. My experience attending this school is satisfactory for the same reason as above.

17. I entered the college/graduate school of Korean medicine because I think Korean medicine doctors $3.44 \pm 1.1$ and the related occupational scope of practice are closely related to my calling.

17-1. My experience attending this school is satisfactory for the same reason as above (or still it is $3.51 \pm 1.0$ closely related to my calling).

18. I entered the college/graduate school of Korean medicine because I or one of my family members $2.18 \pm 1.1$ have/has a chronic disease.

18-1. I am satisfied attending school for the same reasons as above following my experience attending $2.49 \pm 1.2$ the school (the disease can still be a reason to attend school).

19. My family and relatives influenced my decision to attend the college/graduate school of Korean $3.30 \pm 1.2$ medicine.

19-1. My experience attending this school is satisfactory for the same reason as above.

20. My acquaintances (including friends, teachers, and lecturers) influenced my decision to attend the $2.94 \pm 1.2$ college/graduate school of Korean medicine.

20-1. My experience attending this school is satisfactory for the same reason as above.

21. Mass media (including TV programs, the Internet, Korean medicine doctors as role models in the $2.30 \pm 1.1$ media) influenced my decision to attend the college/graduate school of Korean medicine.

21-1. My experience attending this school is satisfactory for the same reason as above.

22. I independently chose to attend the college/graduate school of Korean medicine.

22-1. My experience attending this school is satisfactory for the same reason as above.

23. I am satisfied with my current enrollment in the college/graduate school of Korean medicine.

24. If I were to restart my post-secondary education, I would choose the college/graduate school of $3.74 \pm 1.1$ Korean medicine again.

*number of participants: 420 **the number of participants who answered both question 15 and 15-1 is 202 
The high correlation between questionnaire items and sub-questionnaire items implies a high influence of specific factors that contribute to students' college entrance choices and their current satisfaction with the school they attend. While all items had positive correlations, seven items had high positive correlations at 0.7 or higher. The following items were related to students' positive experiences receiving $\mathrm{KM}$ treatment (item 5, r=0.751), their interest in $\mathrm{KM}$ treatment methods (item 6, $\mathrm{r}=0.731$ ), their enthusiasm for performing clinical skills (item $10, r=0.712$ ), interaction with people who need examinations and treatments (item $12, r=0.809$ ), the ability to learn to perform clinical skills (item $13, r=0.712$ ), the ability to perform psychological treatment (item $14, r=0.719$ ), military duty (item $15, r=0.750$ ), and calling (item 17 , $r=0.796$ ) (Figure 1). Detailed Pearson's correlation coefficients of all questionnaire items and subquestionnaire items are included in Additional File 2 (Additional file 2).

\section{Pearson's correlation between participants' satisfaction, willingness to reenter, and motivation}

The questionnaire items or sub-questionnaire items that showed moderate high correlation with the questionnaire items related to students' satisfaction with their current enrollment were 'currently satisfied because I think Korean medicine doctors are professionals with high social status' (item 1-1, $r=0.5273$ ), 'currently satisfied because I think Korean medicine doctors are professionals with a stable incumbency' (item 2-1, r=0.5622), and 'currently satisfied because I independently chose to attend the college/graduate school of KM' (item 22-1, r=0.6055) (Figure 2).

The questionnaire item or sub-questionnaire item that showed moderate high correlation with the questionnaire item asking whether participants were willing to re-enter the KM college was item 22-1 $(r=0.5273)$. Furthermore, questionnaire items or sub-questionnaire items with a 0.45 or more Pearson's correlation coefficient with the item about re-entry were not only item 1-1 ( $r=0.4514)$ and item 2-1 $(r=0.4873)$, but also items stating 'currently satisfied because I am interested in the theory of KM' (item $10-1, r=0.4712$ ) and 'currently satisfied because I think Korean medicine doctors and the related occupational scope of practice are closely related to my calling' (item 17-1, r=0.489) (Figure 2).

The questionnaire item asking whether college entrance examination scores (college scholastic ability test, high school records) were important factors at the time the students applied to college was the only item that had negative correlations with the questionnaire items about students' current satisfaction $(r=-0.0341)$ and willingness to re-enter ( $r=-0.1269)$, but had low correlation (Figure 2).

\section{Discussion}

\section{Analysis of content validity index}


To interpret proper meaning from responses of questionnaire items, it is important to design a rigorous and high-quality questionnaire [17]. If the questionnaire is poorly designed, it can affect the overall responses and fail to find out essential data from participants [17]. Therefore, we included the content validity test in the process of designing questionnaire.

The content validity experts who were professors gave relatively low scores to questionnaire items including the latent variable of 'requiring personal and clinical skills.' They reported that manual skills are not representative of KM treatment. This decision was made based on their real field experience as KM doctors. Medical practice is not simply related to manual skills, but includes all processes of examination, diagnosis, treatment, and teaching. Therefore, questionnaire items were modified with reference to the field-based medical practices. As a result, when the examples of acupuncture and moxibustion, which are $\mathrm{KM}$ medical practices, were included in the statement, the I-CVI scored much higher.

\section{Analysis of questionnaire scores}

The score of questionnaire items about stable incumbency was high (Mean $\pm S D: 4.08 \pm 0.7$ ) because in South Korea, professionals have relatively high job stability. Questionnaire items about high income also had high scores (Mean \pm SD: $3.93 \pm 0.8$ ) because pay is an important factor when choosing a career. This is aligned with the results of previous studies that pay is a significant factor for medical and dental students in their career choice [7].

The score of the questionnaire regarding students' interest in KM theory was relatively low (Mean \pm SD: $2.99 \pm 1.1$ ). As Korean medicine is a specific major that is taught only after students enter a KM college, it is rare for students to learn KM theory until graduating from high school. The score of this item increased slightly after students entered college. Thus, students may have an increased interest in their major due to the experience of learning KM theory while attending college.

The score of the questionnaire item asking about the treatment method was low due to the lack of experience of learning KM theory (Mean $\pm S D: 2.99 \pm 1.1$ ). The score of its sub-questionnaire slightly increased (Mean $\pm S D: 3.13 \pm 1.0$ ), implying that students developed an interest in KM treatment methods during their education experience.

The score of the questionnaire item concerning students' perception that Korean medicine is based on modern science as an entrance factor was relatively low (Mean \pm SD: 2.65 \pm 1.0 ). As students have not yet learned extensively about KM, they may think that KM adopts inappropriate methodologies and has lowevidence, which in fact contradicts numerous KM research results [26]. The score of the subquestionnaire item slightly increased, but the score was still low (Mean $\pm S D: 2.90 \pm 0.9$ ). The score of the questionnaire item asking whether KM has potential for future development was also low (Mean \pm SD: $3.48 \pm 1.0$ ), and the score of its sub-questionnaire item remained almost unchanged (Mean $\pm S D: 3.40 \pm 0.9$ ).

Cutting-edge systemic biology research takes place in the field of $\mathrm{KM}$, such as network pharmacologybased research. In vitro and in vivo research is also widely conducted [27, 28]. Still, the low scores of such 
questionnaire items (item 6, 6-1, 7, 7-1) imply that the knowledge of KM matriculants is at a superficial level even though they have decided to enter a KM college, as the public strongly insists the necessity of scientificization and technological development of KM, and that herbal medicine has adverse effects [29]. Therefore, scientific research on KM must be promoted more actively to members of the public who show interest in $\mathrm{KM}$, and the results of $\mathrm{KM}$ research must be widely shared with $\mathrm{KM}$ college students.

Only few students consider diverse career choices before their entrance by seeing KM college graduates who take diverse career path, including professors who conduct research on KM. In contrast, the score of the questionnaire item regarding students' willingness to conduct clinical activities was very high (Mean $\pm S D: 4.00 \pm 1.0$ ). Almost every KM doctor conducts clinical activities after their graduation [30]. Therefore, performing clinical activities as a KM doctor is a major factor of entrance. In contrast, the score of the questionnaire item related to the construction of policies and the performance of social activities related to KM was relatively low (Mean $\pm S D: 2.60 \pm 1.0$ ). This can be understood in the same context.

Nonetheless, the scores of the sub-questionnaire items asking about students' willingness to research $\mathrm{KM}$, and policy making and performing social activities related to $\mathrm{KM}$ were slightly increased (Mean $\pm \mathrm{SD}$ : $2.92 \pm 1.0$ and Mean $\pm S D: 2.88 \pm 1.0$, respectively). In contrast, the score of the sub-questionnaire item asking about students' willingness to do clinical activities was slightly decreased (Mean $\pm S D$ : $3.83 \pm 0.9$ ). Many curricular experiences, including lectures, strongly influence students' career decisions [2]. Therefore, while they attend college, students become able to consider different career paths that are not limited to engaging in clinical activities.

The scores of questionnaire items asking about students' interactions with people who need examinations and treatments as an entrance factor, and of learning to perform clinical skills as a KMD were relatively high (Mean \pm SD: $3.62 \pm 1.1$ and Mean \pm SD: $3.85 \pm 1.0$, respectively). Scores of subquestionnaire items (item 12-1, item 13-1) of each questionnaire item did not decrease compared to their related questionnaire items. However, the scores of questionnaire items asking about performing psychological treatments were relatively low compared to former ones. Furthermore, after the entrance, the score of sub-questionnaire items asking about performing psychological treatments was further decreased (Mean \pm SD: $3.44 \pm 1.1$ ). Even though the counseling ability of medical professionals is clearly important, the score of this questionnaire item is low because of the little experience of clinical subjects due to the curriculum of KM colleges and low acknowledgement of psychological treatment, which is actually performed in the KM clinical field [31]. This coincides with previous research that has reported that the young generation considers acupuncture and herbal medicine as the major clinical skills of KM, and that the major area of KM clinical treatment is related to physical health conditions [32], while only $1 \%$ of participants reported that KM could treat psychological and behavioral disorders [33]. This is because the depth of KM college matriculants' knowledge of KM is substantially the same as matriculants of non-KM colleges. Therefore, even though neuropsychiatry is not a major subject of premedical students' educational curriculum, it would be worthwhile to introduce examples of psychological clinical skills and the Department of Korean Neuropsychiatry when teaching about the 
clinical skills of KM. KM college students would then focus more on those issues and will have a wider recognition range of KM clinical skills.

College entrance examinations as college entrance factors reflect realistic aspects of job incumbency rather than job characteristics as medical professionals. Many students want to go to the highest-ranking schools possible with their examination scores. Therefore, examination score becomes a significant factor (Mean \pm SD: $4.03 \pm 0.9$ ).

Mass media had a relatively low effect on students' college entrance choices (Mean $\pm S D: 2.30 \pm 1.1$ ). Many medical students meet a medical role model during their college life [34]. In contrast, premedical students lack this type of experience because of the relatively short time they have attended college. Mass media not only give information regarding college major choices, it can also change students' thoughts about their major choice [35]. However, mass media had a relatively low effect on college entrance choice, and the score of the questionnaire item asking about students' independent choices to attend college was relatively high (Mean \pm SD: $3.85 \pm 1.0$ ). This is because there are relatively few chances to see KM doctors and $\mathrm{KM}$ in the mass media compared to other medical professionals. For this reason, KM should be promoted more actively in the media.

\section{Analysis of correlation between entrance factors at entrance time and present satisfaction}

The greater the correlation between questionnaire items and sub-questionnaire items, the greater the degree to which students' pre-admission perceptions of those factors affect them even after admission. For this reason, the items with high correlation coefficients could be interpreted as factors that have great significance for students' adjustment and satisfaction on current enrollment. These factors should be taken into consideration in students' college major choices.

A positive experience with $\mathrm{KM}$ treatment and interest in $\mathrm{KM}$ treatment methods can be interpreted as items related to the treatment of $\mathrm{KM}$. These experiences could be faced only when they enroll in a KM college. Therefore, the high correlation coefficient of those items can imply that students who had interest in $\mathrm{KM}$ theory, students who had interest during their education in $\mathrm{KM}$ theory and treatment, and those who experienced $\mathrm{KM}$ treatment can be satisfied with attending $\mathrm{KM}$ colleges for those reasons.

The high correlation coefficient of the questionnaire item and sub-questionnaire item about military duty can be interpreted as the same meaning of the high overall score of those items (items 15 and item 15-1). In South Korea, public health doctors can have an alternative role in military duty service system. Students who have not finished their military service may apply at an alternative military duty service and prefer to perform their activities as a KM doctor. Students who have to fulfill their military duty can replace their military duty with public health doctor duty when they have a KMD license; therefore, they seem to be satisfied with their current enrollment.

\section{Analysis of Pearson's correlation between students' satisfaction, willingness to reenter, and questionnaires asking their motivation}


Students dropping out of educational institutions that aim to train and educate medical professionals deplete meaningful personnel resources beyond such students' lack of motivation to become medical professionals [36]. Therefore, it is clearly important to identify the factors that contribute to satisfaction with college enrollment and willingness to re-enter. Students who entered KM colleges due to factors that have high correlation coefficients with satisfaction with the current enrollment could be more satisfied than others.

Factors that affect students' satisfaction with their current enrollment in a KM college are related to social status (item 1-1) and stable incumbency (item 2-1). Students who independently chose to enter college seem to have high satisfaction levels. This high correlation coefficient is a consequence of their independent choice, not of others' opinions. Therefore, such students can be viewed as ones who wished to enter KM colleges before their enrollment.

Students may wish to re-enter a different major because their current major does not fit their vocation or they have a low interest in people [5]. Hence, students who consistently show interest in KMDs' activities related to their job competency have a lower probability of dropping out. Factors related to re-entering the KM colleges can be considered as factors that could bring interest to KMDs' activities related to job competency. Therefore, factors with a high correlation coefficient with the item about re-entering KM colleges may be related to the aptitude for students' major or students' satisfaction.

Theory of KM, interest in clinical activity, and thinking of being a KMD as a calling are closely related to internal factors. Apart from the factor of calling, these factors are closely related to KM doctors' job competency and KM college curriculum. Previous research shows that medical students who enter college because of those internal factors show higher creativity and academic achievement [37]. Therefore, when guiding the college choices of students wishing to go to KM colleges, one should determine the extent to which they want to enter the school due to these internal factors.

The irrelevance of satisfactory level with one's examination score as the important factor corresponds with the results of previous research [18], in which participants who entered KM colleges because of their examination scores had lower satisfaction levels. Because of the characteristics of the College Scholastic Ability Test (CSAT), it is difficult for students to obtain scores in proportion to their interest in specific disciplines, and it is important in high school for students to focus on CSAT preparation [38]. Hence, students who mainly focused on CSAT scores probably selected their college major in reference to the score ranking of the college rather than internal factors, and those who are at the state of high career indecision probably had lower satisfaction with their current major [39]. Therefore, those students have less satisfaction with KM college enrollment.

\section{Limitations and further studies}

Although there are enough participants in this study for statistical analysis, there is always the possibility of gathering more participants. The limitations of this study are that the participation rate of students from different schools was not even. This could be overcome to some extent by delivering web survey 
links in a variety of ways, if further studies were conducted. Focus group interviews were conducted with medical first and second year students rather than premedical second years, and the contents of the questionnaires were modified slightly. Although the positive Pearson's correlation coefficient between questionnaires indicates students' satisfaction with their current enrollment, it may also imply students' rationalization of their choices.

Further research could also be conducted on students who participated in this study at the time of admission, constructing a long-term study that examines changes in KM students' perceptions throughout their academic career. The methodology used in this study can also be adopted in research on other types of medical students.

\section{Conclusion}

Previous research on $\mathrm{KM}$ colleges provided qualitative assessments of educational content and methods but did not examine the factors attributed to KM college entrance. Thus, this study attempted to identify factors that contribute to students' satisfaction in KM colleges. The results of this study may guide students who are suitable for KM college entrance towards KM majors. Additionally, some factors may contribute to the increase of students' current satisfaction in their KM college enrollment. The identification of college entrance factors that are closely related to students' motivation to become KMDs may increase the current academic satisfaction of KM students and decrease drop-out rates. Moreover, some college entrance factors that can be applied to college curriculum or subject teaching, such as interest in research on $\mathrm{KM}$, should be prioritized in the curriculum in the future to increase students' overall satisfaction. Lastly, some responses to the questionnaires indicate that $\mathrm{KM}$ is perceived as an academic discipline. The results show low-scored factors that should be improved through the external promotion of KM.

\section{Abbreviation}

KM: Korean medicine; KMD: Korean medicine doctor; KCD-7: Korean Standard Classification of Disease 7th edition; ICD: International Classification of Diseases

\section{Declarations}

\section{Ethics approval and consent to participate}

This study has been approved by the Public Institutional Bioethics Committee designated by the MOHW (P01-

201903-22-003. All participants could only respond to the online survey if they read the information about the 
contents of survey and gave their informed-consent online before taking part in the survey. The completion of

survey was taken as implied consent.

\section{Consent for publication}

Not applicable

\section{Availability of data and materials}

The datasets used and/or analyzed during the current study are available from the corresponding author on reasonable request.

\section{Competing interest}

The authors declare that they have no competing interests.

\section{Funding}

There was no funding for this study.

\section{Authors' contribution}

HSK and HK planned the study, wrote the protocol, oversaw the study, and contributed to all components. HSK designed the primary questionnaire items, and HMK and UY edited the questionnaire items. HSK and $\mathrm{JL}$ conducted the content validity test and analyzed the data. HMK and UY revised the questionnaire item according to the result of the content validity test. $J L$ conducted the face validity test and edited the questionnaire items based on the results. UY collected the group leaders' contact address and contributed to the message delivery. HSK and JL wrote the manuscript. HK thoroughly revised the manuscript for important intellectual content. All authors read and approved the final manuscript.

\section{Acknowledgements}

We would like to thank Editage (www.editage.co.kr) for English language editing.

\section{Author Details}

${ }^{1}$ Department of Korean Medicine, Dongshin University, 120-9, Dongsindae-gil, Naju-si, Jeollanam-do, Republic of Korea

${ }^{2}$ Dongshin Korean Medicine Hospital, 351, Omok-ro, Yangcheon-gu, Seoul, Republic of Korea

${ }^{3}$ Chung-Yeon Central Institute, 64, Sangmujuangang-ro, Seo-gu, Gwangju, Republic of Korea Author information (supporting information) 
Hyun-Seok Kim:hskimh@kaist.ac.kr; Joohyun Lee: dograngers1542@gmail.com; Hwimun Kim: opqs444@gmail.com; Unho Yang: icariel@naver.com; Hyunho Kim: hyunho.kim@cymedical.org

\section{References}

1. Gerdes $\mathrm{H}$, Mallinckrodt B.: Emotional, social, and academic adjustment of college students: a longitudinal study of retention. Journal of Counseling \& Development 1994, 72(3):281-288.

2. Querido SJ, Vergouw D, Wigersma L, Batenburg RS, De Rond ME, Ten Cate OT: Dynamics of career choice among students in undergraduate medical courses. A BEME systematic review: BEME Guide No. 33. MedicalTteacher 2016, 38(1):18-29.

3. Gibis B, Heinz A, Jacob R, Muller $\mathrm{CH}$ : The career expectations of medical students: findings of a nationwide survey in Germany. Deutsches Arzteblatt International 2012, 109(18):327-332.

4. Hauer KE, Durning SJ, Kernan WN, Fagan MJ, Mintz M, O'Sullivan PS, Battistone M, DeFer T, Elnicki $\mathrm{M}, \mathrm{Harrell} \mathrm{H}$ : Factors associated with medical students' career choices regarding internal medicine. Jama 2008, 300(10):1154-1164.

5. Hyppola H, Kumpusalo E, Neittaanmaki L, Mattila K, Virjo I, Kujala S, Luhtala R, Halila H, Isokoski M: Becoming a doctor-was it the wrong career choice? Social Science \& Medicine 1998, 47(9):13831387.

6. Vaglum P, Wiers-Jenssen J, Ekeberg O: Motivation for medical school: the relationship to gender and specialty preferences in a nationwide sample. Medical Education 1999, 33(4):236-242.

7. Crossley ML, Mubarik A: A comparative investigation of dental and medical student's motivation towards career choice. British Dental Journal 2002, 193(8):471-473.

8. Gallagher J, Clarke W, Wilson N: Understanding the motivation: a qualitative study of dental students' choice of professional career. European Journal of Dental Education: Official Journal of the Association for Dental Education in Europe 2008, 12(2):89-98.

9. Yin CS, Ko SG: Introduction to the history and current status of evidence-based Korean medicine: a unique integrated system of allopathic and holistic medicine. Evidence-Based Complementary and Alternative Medicine: eCAM 2014, 2014:740515.

10. Shin B-C, Kim S, Cho Y-H: Syndrome pattern and its application in parallel randomized controlled trials. Chinese Journal of Integrative Medicine 2013, 19(3):163-171.

11. Han SY, Kim HY, Lim JH, Cheon J, Kwon YK, Kim H, Yang GY, Chae H: The past, present, and future of traditional medicine education in Korea. Integrative Medicine Research 2016, 5(2):73-82.

12. Kim BS: Concept of talent on the doctor of Korean medicine to pursue university education in vocational performed for Korean medical doctor. Journal of Physiology \& Pathology in Korean Medicine 2015, 29(3):256-266.

13. Lee JH, Kim BS: Study of Korean medicines education in accordance with curriculum part of world federation medical educaition global standards. Journal of Korean Medicine 2018, 39(3):28-40. 
14. Kim H, Jeong H, Jeon P, Kim S, Park YB, Kang Y: Perception study of traditional Korean medical students on the medical education using the Dundee ready educational environment measure. Evidence-Based Complementary and Alternative Medicine: eCAM 2016, 2016:6042967.

15. Leem J, Kim Y, Heo J, Jung M, Kim S, Lee H, Park JH, Kim T-H: Experiences, perceptions and needs on the education of evidence-based medicine among Korean mdicine college students: a nationwide online survey. European Journal of Integrative Medicine 2017, 15:10-16.

16. Cha H-Y, Kim N-H, Hong J-W, Shin S-W: Evaluation of the implementation of problem-based learning in Korean medical education. Journal of Physiology \& Pathology in Korean Medicine 2012, 26:351359.

17. Artino Jr AR, La Rochelle JS, Dezee KJ, Gehlbach H: Developing questionnaires for educational research: AMEE Guide No. 87. Medical Teacher 2014, 36(6):463-474.

18. Kwon S-W, Shin S-W, Lim B-M: A survey of students' satisfaction with education in traditional Korean medicine. The Journal of Korean Medicine 2012, 33(1):1-11.

19. Rubio DM, Berg-Weger M, Tebb SS, Lee ES, Rauch S: Objectifying content validity: conducting a content validity study in social work research. Social Work Research 2003, 27(2):94-104.

20. Polit DF, Beck CT: The content validity index: are you sure you know what's being reported? Critique and recommendations. Research in Nursing \& Health 2006, 29(5):489-497.

21. McKenzie JF, Wood ML, Kotecki JE, Clark JK, Brey RA: Establishing content validity: using qualitative and quantitative steps. American Journal of Health Behavior 1999, 23(4):311-318.

22. Nevo B: Face validity revisited. Journal of Educational Measurement 1985, 22(4):287-293.

23. Huang KT, Owino C, Vreeman RC, Hagembe M, Njuguna F, Strother RM, Gramelspacher GP: Assessment of the face validity of two pain scales in Kenya: a validation study using cognitive interviewing. BMC Palliative Care 2012, 11(1):5.

24. Karabenick SA, Woolley ME, Friedel JM, Ammon BV, Blazevski J, Bonney CR, GROOT ED, Gilbert MC, Musu L, Kempler TM: Cognitive processing of self-report items in educational research: do they think what we mean? Educational Psychologist 2007, 42(3):139-151.

25. Committee YBoTKMP: 2017 Year book of traditional Korean medicine. Daejeon: Kumkang Printing, Pusan National University School of Korean Medicine, Korea Institute of Oriental Medicine, The Association of Korean Medicine; 2019.

26. Kim S, Choi S, Lee S, Lee J-H, Park JJ: Case study projects by a Korean national research agency: past 12 years and future. Complementary Therapies in Clinical Practice 2019, 35:48-52.

27. Lee S: Systems biology-a pivotal research methodology for understanding the mechanisms of traditional medicine. Journal of Pharmacopuncture 2015, 18(3):11.

28. Lee W-Y, Lee C-Y, Kim Y-S, Kim C-E: The methodological trends of traditional herbal medicine employing network pharmacology. Biomolecules 2019, 9(8):362.

29. Hyun MK: The needs and priorities for government grants for traditional Korean medicine: comparing the public and traditional Korean medicine doctors. Evidence-Based Complementary and Alternative 
Medicine 2016, 2016.

30. Bak Y-H, Huang D-S, Shin H-K: A survey of the medical treatment environment in traditional Korean medicine clinics. The Journal of Korean Medicine 2011, 32.

31. Kim KH, Jang S, Lee JA, Jang B-H, Go H-Y, Park S, Jo H-G, Lee MS, Ko S-G: Experiences providing medical assistance during the Sewol ferry disaster using traditional Korean medicine. EvidenceBased Complementary and Alternative Medicine 2017, 2017.

32. Lee J-W, Kim G-C: Survey of university students' perception degree of Korean medical care. The Journal of the Korea Institute of Oriental Medical Diagnostics 2012, 16(2):47-58.

33. Park J-K, Yim J-H, Kim H, Park Y-J, Park Y-B: Perception and utilization of Korean medicine of the university students in Seoul: a survey research. The Journal of the Korea Institute of Oriental Medical Diagnostics 2014, 18(3):187-204.

34. Wright S, Wong A, Newill C: The impact of role models on medical students. Journal of General Internal Medicine 1997, 12(1):53-56.

35. Saleem N, Mian A, Saleem HI, Rao MS: Career selection: role of parent's profession mass media and ersonal choice. Bulletin of Education and Research 2014, 36(2).

36. Baars G, Arnold I: Early identification and characterization of students who drop out in the first year at university. Journal of College Student Retention: Research, Theory \& Practice 2014, 16(1):95-109.

37. Sobral DT: What kind of motivation drives medical students' learning quests? Medical Education 2004, 38(9):950-957.

38. Sovet L, Park MS-A, Jung S: Validation and psychometric properties of academic major satisfaction scale among Korean college students. Social Indicators Research 2014, 119(2):1121-1131.

39. Kang C: University prestige and choice of major field: evidence from South Korea. In: Far Eastern Meeting for Econometric Society, Seoul, South Korea: 2004; 2004.

\section{Additional Files}

Additional file 1: Questionnaire used at the survey. (DOCX 21kb)

Additional file 2: Basic characteristics of respondents ( $\mathrm{N}=420)$. (DOCX 14kb)

Additional file 3: Pearson's Correlation between Questionnaire Items and Their Sub-questionnaire Items. (DOCX 17kb)

\section{Figures}




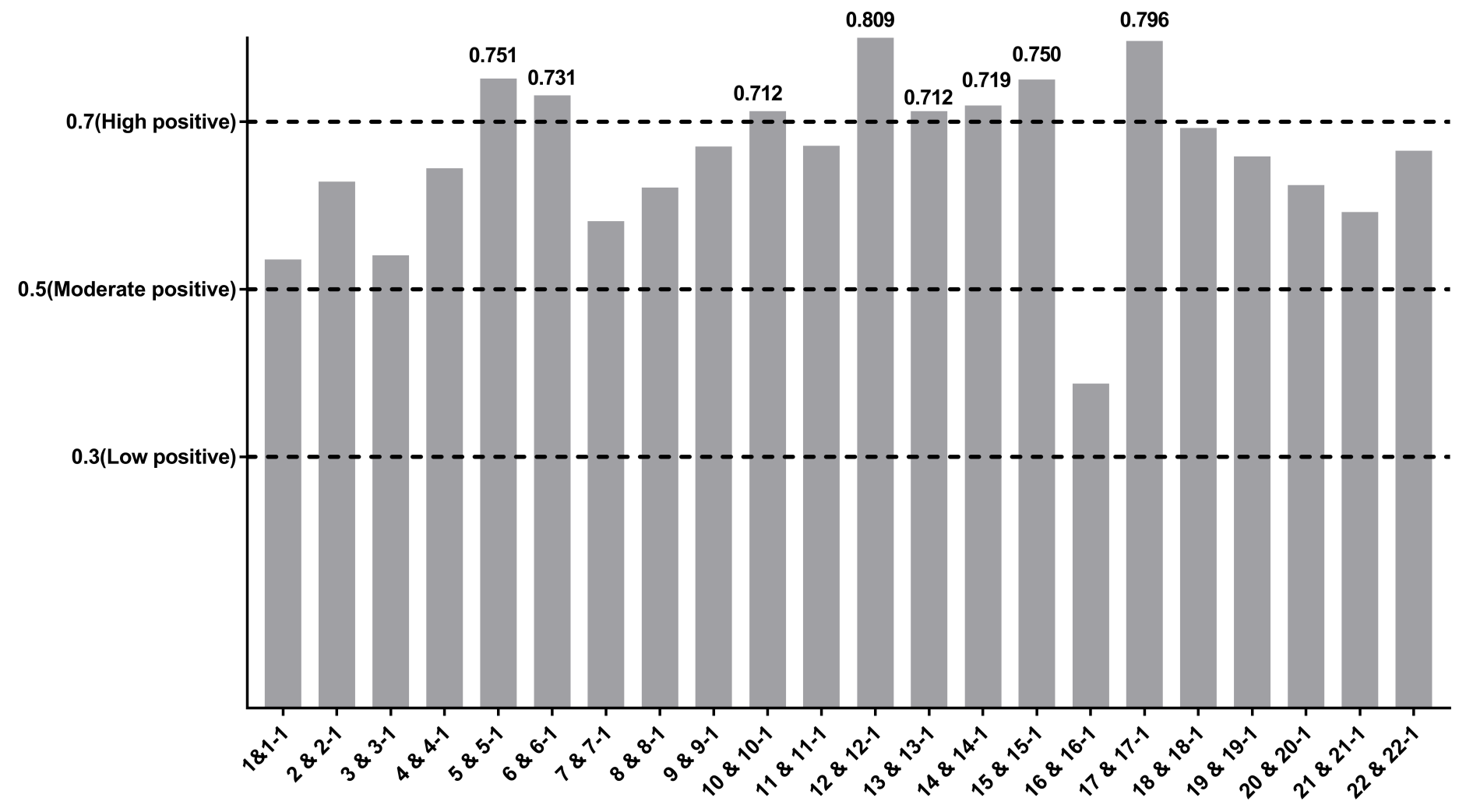

Figure 1

Pearson's correlation coefficient between questionnaire items and sub-questionnaire items. Among 22 items, 5 items had high positive correlation coefficient $(r \geq 0.7)$. 


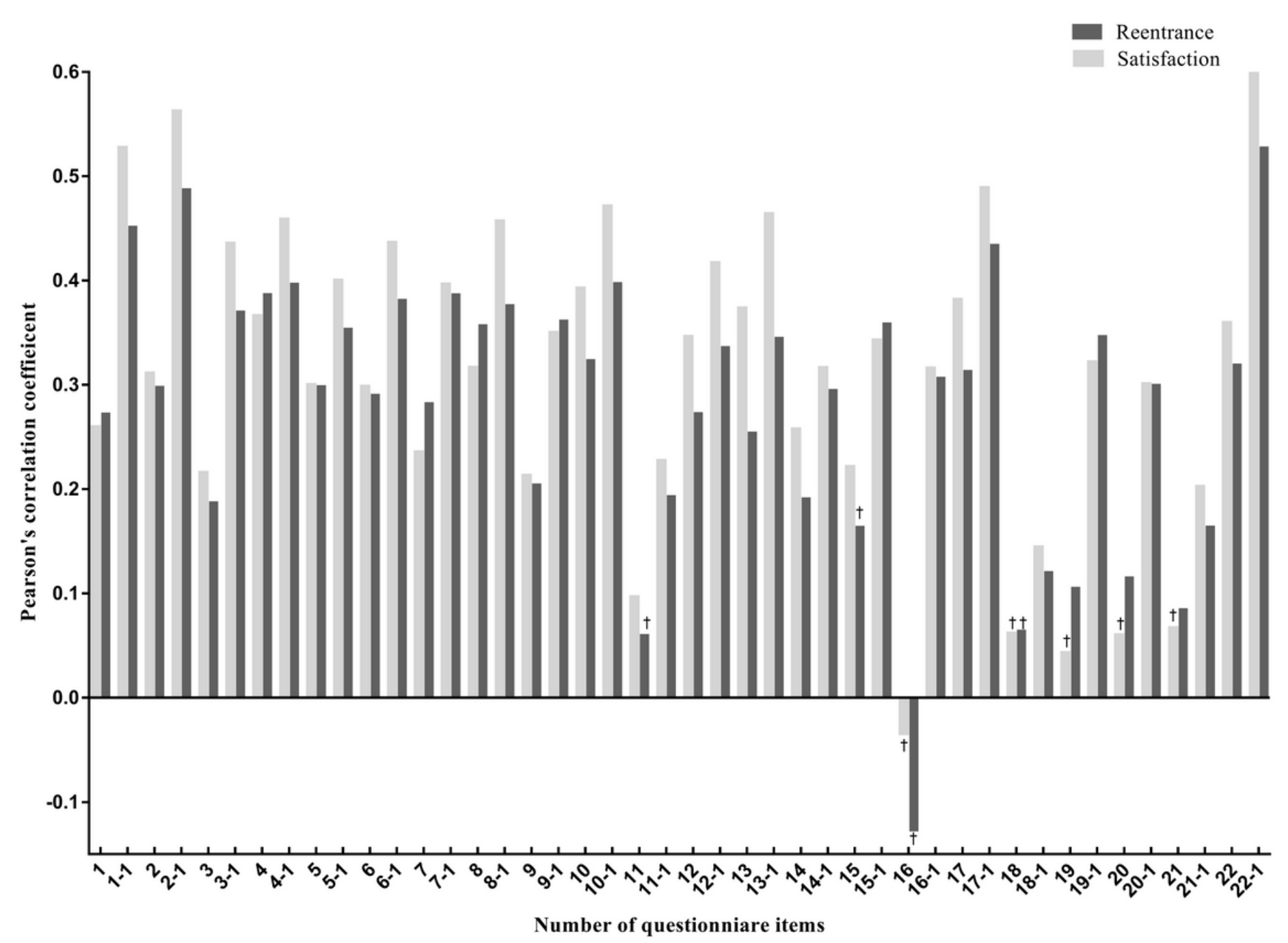

Figure 2

Figure 2. Pearson's correlation coefficient between questionnaire and sub-questionnaire items and items concerning students' satisfaction with their current enrollment (item 23), and questionnaire and subquestionnaire items and items asking whether participants were willing to re-enter the KM college (item 24). + : correlation statistically insignificant $(p>0.005)$

\section{Supplementary Files}

This is a list of supplementary files associated with this preprint. Click to download.

- AdditionalFile3.docx

- AdditionalFile1.docx

- AdditionalFile2.docx 